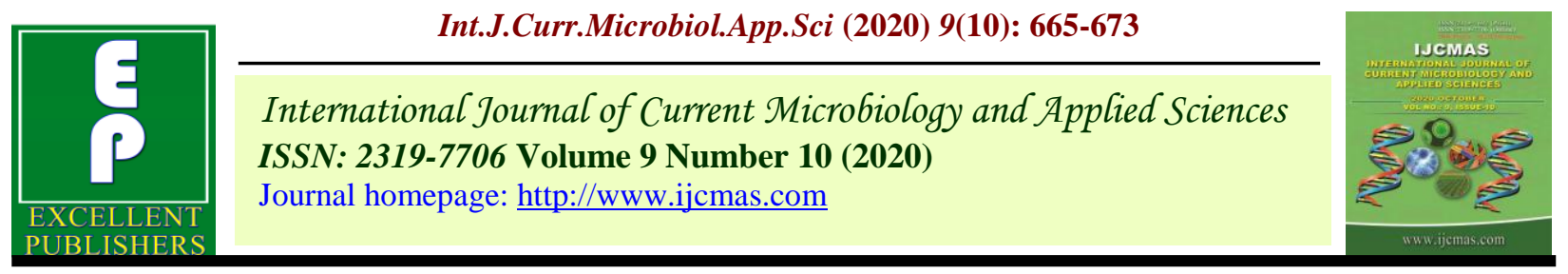

Original Research Article

https://doi.org/10.20546/ijcmas.2020.910.080

\title{
Combining ability Analysis for Yield and Yield Components in Okra [Abelmoschus esculentus (L.) Moench]
}

\author{
H. K. Koli ${ }^{1}$ and A. I. Patel ${ }^{2 *}$ \\ ${ }^{1}$ Genetics and Plant Breeding, ${ }^{2}$ ASPEE College of Horticulture and Forestry, NMCA, NAU, \\ Navsari, Gujarat, India \\ *Corresponding author
}

\begin{tabular}{|c|c|}
\hline & A B S T R A C T \\
\hline $\begin{array}{l}\text { Okra, } \\
\text { Abelmoschus } \\
\text { esculentus }\end{array}$ & \multirow{3}{*}{$\begin{array}{l}\text { Information on magnitude of combining ability were obtained for fruit yield and } \\
\text { its related components; involving eight parents and their } 28 \mathrm{~F}_{1} \text { hybrids (excluding } \\
\text { reciprocal cross) along with a commercial hybrid check 'GJOH-4' of okra in } \\
\text { randomized block design with three replications. The magnitude of } s c a \text { variance } \\
\text { was higher than } g c a \text { variance for all the traits revealed preponderance of non- } \\
\text { additive gene action for all the traits. The estimation of } g c a \text { effects indicated that } \\
\text { parents viz., Arka Abhay and NOL-18-14 were good general combiner for fruit } \\
\text { yield and its related traits. The estimation of sca effects indicated that hybrids, viz., } \\
\text { Arka abhay x GAO-5, Arka abhay x NOL-18-14 and GAO-5 x NOL-18-14 were } \\
\text { exhibited high heterosis coupled with high } s c a \text { effects also. }\end{array}$} \\
\hline Article Info & \\
\hline $\begin{array}{l}\text { Accepted: } \\
\text { 07 September } 2020 \\
\text { Available Online: } \\
10 \text { October } 2020\end{array}$ & \\
\hline
\end{tabular}

\section{Introduction}

Okra [Abelmoschus esculentus (L.) Moench] is an important fruit vegetable crop cultivated in various states of India. Several species of the genus Abelmoschus are grown in many parts of the world among them Abelmoschus esculentus is most commonly cultivated in Asia and has a great commercial demand due to its nutritional values. In India area, production and productivity of okra in year 2018-19 is 511 ('000 ha), 62.19 lakh tonnes and 12.17 MT/hectares, respectively (Anon., 2019). Combining ability is referred as an ability of a parent to transmit its performance to its off springs. As the combining ability often depends upon complex interaction systems among genes, certain combinations nick well to produce superior off springs, whereas, others involving equally promising parents produce disappointing progeny. The concept of combining ability was initially developed by Sprague and Tatum in 1942. Combining ability indicates capacity of individual parents to transmit superior performances to its off springs. General combining ability $(g c a)$ is the average performance of a parent in a series of crosses and specific combining ability (sca) is an average performance and reflections of those cases in which certain combinations do relatively better or worse than would be expected on the basis of average performance of line involved. Sprague and Tatum (1942) 
revealed that $g c a$ is basically due to additive effects of gene, whereas sca is due to interallelic interactions. Diallel analysis is the quickest method of understanding the genetic nature of polygenic characters and it also helps to ascertain the prepotency of parents.

\section{Materials and Methods}

The present study consists of 8 different okra genotypes viz., Arka Abhay, GOA-5, NOL18-9, NOL-18-12, NOL-18-14, NOL-18-15, NOL-18-16, and NOL-18-17. The parents were crossed in half diallel mating design excluding reciprocals to produce 28 hybrids in summer 2019. The $\mathrm{F}_{1}$ hybrids were obtained were evaluated at College farm, N. M. College of Agriculture, N. A. U., Navsari in kharif-2019, with three replications using okra hybrid GJOH-4 as standard check. Each and every genotype was grown in single line using $60 \mathrm{~cm} \times 30 \mathrm{~cm}$ spacing. The different 11 quantitative characters like days to $50 \%$ flowering, plant height $(\mathrm{cm})$, branches per plant, fruit weight $(\mathrm{g})$, fruit length $(\mathrm{g})$, fruit diameter $(\mathrm{cm})$, fruit per plant, fruit yield per plant, internodal length $(\mathrm{cm})$, number of seeds per fruit, 100 seed weight (g) has been recorded. Data was analyzed as per Griffing's Method-II and Model-I (fixed-effect model) which involves direct crosses and parents.

\section{Results and Discussion}

The analysis of variance for combining ability for different traits was presented in Table 1. The analysis of variance for combining ability revealed that mean sum of square due to general combining ability and specific combining ability were significant for all the characters under study. It revealed that involvement of both additive as well as nonadditive gene action responsible for inheritance of fruit yield and yield related traits in okra.
Similar result was reported by Patil et al., (2016), Tiwari et al., (2016), Wakode et al., (2016), Devi et al., (2017), Paul et al., (2017), Rameshkumar et al., (2017) and Lokeswari et al., (2018). However, the magnitudes of variance $g c a$ were lower than variance $s c a$ for all the traits indicating the predominance of the non-additive gene action, for all the traits.

The estimates of general combining ability effects for each parent are presented in table 2. In the present investigation, Arka Abhay and NOL-18-14 were good general combiner for fruit per plant, internodal length as well as branches per plant; NOL-18-14 was for fruit yield per plant and fruit weight; Arka Abhay, GAO-5 and NOL-18-14 were for plant height; NOL-18-12 and NOL-18-14 were for fruit length; GAO-5 and NOL-18-14 were for fruit diameter, Arka Abhay was for days to $50 \%$ flowering; NOL18-9 and NOL-18-17 were for number of seed per fruit while NOL-18-9, NOL-18-16 and NOL-18-17 were for 100 seed weight. Similar result was reported by Patel et al., (2015b), Kumar et al., (2016), Tiwari et al., (2016), Lyngdoh et al., (2017), More et al., (2017), Satish et al., (2017), Hadiya et al., (2018), Makdoomi et al., (2018), Demta and Surin (2019), and Pithiya et al., (2020) in okra.

Specific combining ability effect helps in identification of superior cross combination (good specific combiners) for commercial exploitation of heterosis. In present investigation, eight hybrids viz., NOL-18-12 x NOL-18-15, NOL-18-19 x NOL-18-16, Arka Abhay x GAO-5, NOL-18-15 x NOL-18-16, NOL-18-15 x NOL-18-17, Arka Abhay $x$ NOL-18-14, GAO-5 x NOL-18-14, GAO-5 x NOL-18-12 were found significant and positive sca effects for fruit yield per plant. The estimates of sca effects (Table-3) revealed that none of the hybrid exhibited consistently significant and desirable sca effects for all the characters. 
Table.1 Analysis of variance (MSS) for combining ability in respect to various characters in okra

\begin{tabular}{|c|c|c|c|c|c|c|c|c|c|c|c|c|c|}
\hline $\begin{array}{l}\text { Sr. } \\
\text { No. }\end{array}$ & $\begin{array}{c}\text { Sources } \\
\text { of } \\
\text { variation }\end{array}$ & DF & $\begin{array}{c}\text { Days to } \\
50 \% \\
\text { flowering }\end{array}$ & $\begin{array}{l}\text { Plant } \\
\text { height } \\
(\mathrm{cm})\end{array}$ & $\begin{array}{l}\text { Branches } \\
\text { per plant }\end{array}$ & $\begin{array}{c}\text { Fruit } \\
\text { weight } \\
\text { (g) }\end{array}$ & $\begin{array}{c}\text { Fruit } \\
\text { length } \\
(\mathbf{c m})\end{array}$ & $\begin{array}{c}\text { Fruit } \\
\text { diameter } \\
(\mathbf{c m})\end{array}$ & $\begin{array}{c}\text { Fruits } \\
\text { per } \\
\text { plant }\end{array}$ & $\begin{array}{c}\text { Fruit } \\
\text { yield per } \\
\text { plant (g) }\end{array}$ & $\begin{array}{l}\text { Internodal } \\
\text { length }(\mathrm{cm})\end{array}$ & $\begin{array}{c}\text { Number } \\
\text { of seeds } \\
\text { per } \\
\text { fruit }\end{array}$ & $\begin{array}{c}100 \text { seed } \\
\text { weight } \\
\text { (g) }\end{array}$ \\
\hline 1 & $g c a$ & 7 & $3.15^{*}$ & $350.13 * *$ & $0.38 * *$ & $2.91 * *$ & $5.43 * *$ & $0.02 * *$ & $6.76^{* *}$ & $3788.16 * *$ & $2.04 * *$ & $43.50 * *$ & $0.52 * *$ \\
\hline 2 & sca & 28 & $2.70 * *$ & $92.99 * *$ & $0.13 * *$ & $1.16^{* *}$ & $1.16^{* *}$ & $0.08 * *$ & $2.11 * *$ & $786.42 * *$ & $0.98 * *$ & $28.82 * *$ & $0.16^{* *}$ \\
\hline 3 & Error & 70 & 1.14 & 10.48 & 0.03 & 0.30 & 0.27 & 0.002 & 0.30 & 114.84 & 0.14 & 6.97 & 0.03 \\
\hline 4 & $\sigma_{\text {gca }}^{2}$ & - & 0.20 & 33.97 & 0.04 & 0.26 & 0.52 & 0.001 & 0.65 & 367.33 & 0.19 & 3.65 & 0.04 \\
\hline 5 & $\sigma_{\text {sca }}^{2}$ & - & 1.56 & 82.51 & 0.10 & 0.86 & 0.89 & 0.005 & 1.81 & 671.58 & 0.83 & 21.85 & 0.13 \\
\hline 6 & $\begin{array}{c}\sigma_{\mathrm{gca}}^{2} / \\
\sigma_{\mathrm{sca}}^{2}\end{array}$ & - & 0.13 & 0.41 & 0.34 & 0.30 & 0.58 & 0.20 & 0.36 & 0.55 & 0.23 & 0.17 & 0.37 \\
\hline
\end{tabular}

$*$ - Significant at $5 \%$ and $* *$ - Significant at $1 \%$

Table.2 General combining ability effects for different characters in okra

\begin{tabular}{|c|c|c|c|c|c|c|c|c|c|c|c|c|}
\hline $\begin{array}{l}\text { Sr. } \\
\text { No. }\end{array}$ & Parents & $\begin{array}{c}\text { Days to } \\
50 \% \\
\text { flowering }\end{array}$ & $\begin{array}{c}\text { Plant } \\
\text { height } \\
(\mathrm{cm})\end{array}$ & $\begin{array}{l}\text { Branches } \\
\text { per plant }\end{array}$ & $\begin{array}{c}\text { Fruit } \\
\text { weight } \\
\text { (g) }\end{array}$ & $\begin{array}{c}\text { Fruit } \\
\text { length } \\
(\mathrm{cm})\end{array}$ & $\begin{array}{c}\text { Fruit } \\
\text { diameter } \\
(\mathrm{cm})\end{array}$ & $\begin{array}{c}\text { Fruits } \\
\text { Per } \\
\text { plant }\end{array}$ & $\begin{array}{c}\text { Fruit } \\
\text { yield } \\
\text { per plant } \\
\text { (g) }\end{array}$ & $\begin{array}{l}\text { Internodal } \\
\text { length } \\
\text { (cm) }\end{array}$ & $\begin{array}{c}\text { Number } \\
\text { of } \\
\text { seeds } \\
\text { per fruit }\end{array}$ & $\begin{array}{c}100 \text { seed } \\
\text { Weight } \\
\text { (g) }\end{array}$ \\
\hline 1. & Arka Abhay & $-0.66^{*}$ & $6.13 * *$ & $0.21 * *$ & 0.003 & $-0.58 * *$ & 0.03 & $0.41 *$ & 3.49 & $-0.32 * *$ & 0.57 & 0.10 \\
\hline 2. & GAO -5 & -0.23 & $3.84 * *$ & 0.08 & 0.10 & $-0.34 *$ & $0.04 *$ & 0.19 & 5.76 & $0.36^{* *}$ & 1.31 & $0.12 *$ \\
\hline 3. & NOL-18-9 & 0.44 & $-4.53 * *$ & -0.10 & 0.06 & $-0.95 * *$ & -0.01 & $-0.64 * *$ & $-8.96 * *$ & $0.34 * *$ & $-1.67 *$ & $-0.39 * *$ \\
\hline 4. & NOL-18-12 & -0.19 & $-1.06 * *$ & 0.02 & 0.09 & $0.51 * *$ & $-0.04 *$ & 0.23 & 2.84 & -0.07 & -0.89 & 0.10 \\
\hline 5. & NOL-18-14 & -0.56 & $9.71 * *$ & $0.33 * *$ & $1.14 * *$ & $1.46^{* *}$ & $0.06^{* *}$ & $1.69 * *$ & $42.21 * *$ & $-0.81 * *$ & $4.09 * *$ & $0.32 * *$ \\
\hline 6. & NOL-18-15 & 0.28 & $-4.13 * *$ & $-0.17 * *$ & $-0.70 * *$ & -0.06 & $-0.04 *$ & $-0.79 * *$ & $-19.25 * *$ & 0.03 & -0.62 & $0.11 *$ \\
\hline 7. & NOL-18-16 & -0.13 & $-7.25 * *$ & $-0.23^{* *}$ & $-0.40^{*}$ & 0.16 & 0.01 & $-0.65 * *$ & $-16.65 * *$ & -0.15 & -0.02 & $-0.19 * *$ \\
\hline \multirow[t]{2}{*}{8.} & NOL-18-17 & $1.04 * *$ & $-2.70 * *$ & $-0.14 * *$ & -0.29 & -0.21 & $-0.05 * *$ & $-0.44 * *$ & $-9.44 * *$ & $0.63 * *$ & $-2.77 * *$ & $-0.17 * *$ \\
\hline & S. E. $\left(g_{i}\right) \pm$ & 0.32 & 0.96 & 0.05 & 0.16 & 0.15 & 0.02 & 0.16 & 3.17 & 0.11 & 0.78 & 0.05 \\
\hline
\end{tabular}

* - Significant at $5 \%$ and **-Significant at $1 \%$ 
Table.3 Specific combining ability effects for different characters in okra

\begin{tabular}{|c|c|c|c|c|c|c|c|c|c|c|c|c|}
\hline $\begin{array}{l}\text { Sr. } \\
\text { No. }\end{array}$ & Hybrids & $\begin{array}{c}\text { Days to } \\
50 \% \\
\text { flowering }\end{array}$ & $\begin{array}{c}\text { Plant } \\
\text { height } \\
\text { (cm) }\end{array}$ & $\begin{array}{c}\text { Branch } \\
\text { es per } \\
\text { plant }\end{array}$ & $\begin{array}{c}\text { Fruit } \\
\text { weight } \\
\text { (g) }\end{array}$ & $\begin{array}{l}\text { Fruit } \\
\text { length } \\
\text { (cm) }\end{array}$ & $\begin{array}{c}\text { Fruit } \\
\text { diamete } \\
\text { r (cm) }\end{array}$ & $\begin{array}{c}\text { Fruits per } \\
\text { plant }\end{array}$ & $\begin{array}{c}\text { Fruit } \\
\text { yield per } \\
\text { plant (g) }\end{array}$ & $\begin{array}{l}\text { Internod } \\
\text { al length } \\
(\mathrm{cm})\end{array}$ & $\begin{array}{c}\text { Number } \\
\text { of seeds } \\
\text { per fruit }\end{array}$ & $\begin{array}{c}100 \text { seed } \\
\text { weight } \\
\text { (g) }\end{array}$ \\
\hline 1. & Arka Abhay x GAO-5 & -1.14 & $15.16^{* *}$ & $0.50 * *$ & 0.99 & 0.66 & $0.14^{* *}$ & $1.05^{*}$ & $32.34 * *$ & $-2.31 * *$ & $9.03 * *$ & $0.37 *$ \\
\hline 2. & Arka Abhay x NOL-18-9 & 0.52 & $10.57 * *$ & 0.14 & 0.36 & 0.00 & 0.03 & 0.47 & 3.69 & -0.12 & -2.79 & $-0.35^{*}$ \\
\hline 3. & Arka Abhay x NOL-18-12 & -1.18 & -3.67 & -0.04 & -0.27 & -0.49 & -0.10 & $-1.26^{*}$ & $-24.94 *$ & $0.96 * *$ & -1.10 & $0.33 *$ \\
\hline 4. & Arka Abhay x NOL-18-14 & 0.86 & 5.40 & -0.15 & $1.02 *$ & 0.58 & 0.09 & $1.29 *$ & $27.72 * *$ & 0.01 & 0.92 & -0.39 \\
\hline 5. & Arka Abhay x NOL-18-15 & $2.36^{*}$ & $-19.90 * *$ & $-0.39 *$ & -0.93 & -0.77 & $-0.15^{* *}$ & $-1.11 *$ & $-27.70 * *$ & $1.15 * *$ & $-5.90 *$ & -0.25 \\
\hline 6. & Arka Abhay x NOL-18-16 & -1.91 & -1.61 & -0.26 & 0.67 & -0.05 & -0.09 & $-2.18 * *$ & $-26.03 *$ & $0.77 *$ & -0.84 & 0.32 \\
\hline 7. & Arka Abhay x NOL-18-17 & -0.74 & 4.34 & -0.01 & -0.57 & 0.20 & 0.03 & 0.75 & 10.33 & 0.36 & $8.32 * *$ & 0.13 \\
\hline 8. & GAO-5 x NOL-18-9 & 0.42 & $-11.78 * *$ & $-0.46 * *$ & -0.79 & -0.73 & -0.05 & $-1.11 *$ & -14.71 & $1.57 * *$ & $-5.14 *$ & $-0.38 *$ \\
\hline 9. & GAO-5 x NOL-18-12 & -1.28 & $7.88^{*}$ & 0.16 & $1.24 *$ & $1.10^{*}$ & 0.05 & 0.69 & $25.26 *$ & $0.98 * *$ & 1.82 & -0.03 \\
\hline 10. & GAO-5 x NOL-18-14 & -1.58 & 5.12 & $0.39 *$ & $1.25 *$ & -0.53 & -0.09 & 0.70 & $26.69 *$ & 0.18 & -0.83 & 0.29 \\
\hline 11. & GAO-5 x NOL-18-15 & -0.74 & -4.18 & 0.08 & $-1.12 *$ & 0.04 & $-0.14 * *$ & -0.09 & $-20.59 *$ & 0.11 & $-8.92 * *$ & 0.26 \\
\hline 12. & GAO-5 x NOL-18-16 & -0.68 & -5.13 & 0.01 & -0.06 & 0.59 & 0.03 & $-1.97 * *$ & $-29.43 * *$ & $1.30 * *$ & -0.98 & $0.66^{* *}$ \\
\hline 13. & GAO-5 x NOL-18-17 & 0.16 & $-12.64 * *$ & $-0.35^{*}$ & $-1.32 *$ & $-0.99 *$ & -0.05 & -0.17 & $-27.50 * *$ & $1.22 * *$ & 0.44 & $-0.59 * *$ \\
\hline 14. & NOL-18-9 x NOL-18-12 & 1.39 & 2.32 & $0.33 *$ & 0.06 & -0.15 & $0.13^{*}$ & $1.78 * *$ & 19.84 & $-0.87 *$ & -0.26 & $0.84 * *$ \\
\hline 15. & NOL-18-9 x NOL-18-14 & 1.42 & -3.24 & 0.16 & 0.04 & 0.95 & -0.08 & $-1.81 * *$ & -17.10 & -0.02 & 1.42 & $-0.34^{*}$ \\
\hline 16. & NOL-18-9 x NOL-18-15 & 0.59 & $-12.04 * *$ & $-0.48 * *$ & $-1.13 *$ & -0.10 & -0.05 & $-2.00 * *$ & $-28.34 * *$ & 0.23 & -3.60 & -0.04 \\
\hline 17. & NOL-18-9 x NOL-18-16 & -1.68 & 3.28 & $0.51 * *$ & 0.37 & $1.41 * *$ & 0.05 & $2.79 * *$ & $36.69 * *$ & -0.17 & 4.40 & 0.23 \\
\hline 18. & NOL-18-9 x NOL-18-17 & -0.18 & $-9.23 * *$ & $-0.44 * *$ & $-1.52 * *$ & $-1.04 *$ & -0.05 & $-1.68 * *$ & $-41.75^{* *}$ & $1.07 * *$ & -2.78 & -0.09 \\
\hline 19. & NOL-18-12 x NOL-18-14 & $-3.28 * *$ & 4.55 & 0.11 & -0.03 & -0.17 & 0.07 & 0.99 & 16.24 & -0.59 & $8.04 * *$ & 0.07 \\
\hline 20. & NOL-18-12 x NOL-18-15 & -1.44 & 5.29 & $0.34 *$ & 0.80 & 0.94 & 0.04 & $2.00 * *$ & $37.49 * *$ & -0.14 & $9.02 * *$ & $0.41 *$ \\
\hline 21. & NOL-18-12 x NOL-18-16 & $2.29 *$ & $-13.59 * *$ & $-0.40 *$ & $-1.44 * *$ & $-1.73 * *$ & -0.03 & -0.01 & $-23.84 *$ & $-0.71 *$ & -3.18 & $-0.79 * *$ \\
\hline 22. & NOL-18-12 x NOL-18-17 & 0.12 & $8.06^{*}$ & $0.38 *$ & $1.34 *$ & $2.26^{* *}$ & 0.01 & -0.81 & 14.92 & $-1.22 * *$ & $-5.36^{*}$ & $-0.34^{*}$ \\
\hline 23. & NOL-18-14 x NOL-18-15 & -0.74 & 2.19 & $0.50 * *$ & 0.64 & 0.23 & 0.02 & 0.68 & 18.05 & -0.52 & 3.10 & 0.13 \\
\hline 24. & NOL-18-14 x NOL-18-16 & -0.68 & $-16.99 * *$ & $-0.57 * *$ & $-1.89 * *$ & 0.24 & 0.00 & $-1.33^{*}$ & $-42.51 * *$ & 0.68 & -3.70 & 0.00 \\
\hline
\end{tabular}


Table.3 Conti...

\begin{tabular}{|c|c|c|c|c|c|c|c|c|c|c|c|c|}
\hline $\begin{array}{l}\text { Sr. } \\
\text { No. }\end{array}$ & Hybrids & $\begin{array}{c}\text { Days to } \\
50 \% \\
\text { flowering }\end{array}$ & $\begin{array}{c}\text { Plant } \\
\text { height } \\
(\mathbf{c m})\end{array}$ & $\begin{array}{l}\text { Branches } \\
\text { per plant }\end{array}$ & $\begin{array}{c}\text { Fruit } \\
\text { weight } \\
\text { (g) }\end{array}$ & $\begin{array}{c}\text { Fruit } \\
\text { length } \\
(\mathbf{c m})\end{array}$ & $\begin{array}{c}\text { Fruit } \\
\text { diamete } \\
\text { r (cm) }\end{array}$ & $\begin{array}{c}\text { Fruits } \\
\text { per } \\
\text { plant }\end{array}$ & $\begin{array}{c}\text { Fruit } \\
\text { yield per } \\
\text { plant (g) }\end{array}$ & $\begin{array}{l}\text { Internoda } \\
\text { I length } \\
(\mathrm{cm})\end{array}$ & $\begin{array}{l}\text { Number } \\
\text { of seeds } \\
\text { per fruit }\end{array}$ & $\begin{array}{c}100 \text { seed } \\
\text { weight } \\
\text { (g) }\end{array}$ \\
\hline 25. & NOL-18-14 x NOL-18-17 & $2.49 *$ & -0.60 & 0.07 & 0.33 & $1.39 * *$ & $-0.12 *$ & 0.93 & 14.11 & -0.63 & -4.14 & $0.34 *$ \\
\hline 26. & NOL-18-15 x NOL-18-16 & $-2.18 *$ & $10.01 * *$ & -0.08 & 0.59 & 0.62 & $0.11 *$ & $2.08 * *$ & $31.47 * *$ & $-1.19 * *$ & $9.55^{* *}$ & $-0.70^{* *}$ \\
\hline 27. & NOL-18-15 x NOL-18-17 & -0.01 & $8.63 * *$ & 0.30 & $2.21 * *$ & $1.95 * *$ & 0.08 & 0.67 & $29.47 * *$ & $-0.98 * *$ & -2.76 & -0.12 \\
\hline \multirow[t]{2}{*}{28.} & NOL-18-16 x NOL-18-17 & -0.28 & -0.71 & -0.24 & 0.81 & $-1.51 * *$ & $-0.14 * *$ & -0.87 & -2.63 & -0.17 & $-5.70^{*}$ & 0.08 \\
\hline & S. E. $\left(s_{i j}\right) \pm$ & 0.97 & 2.94 & 0.15 & 0.50 & 0.47 & 0.05 & 0.50 & 9.72 & 0.34 & 2.39 & 0.16 \\
\hline
\end{tabular}

* - Significant at $5 \%$ and **-Significant at $1 \%$ 
Table.4 Best general combiners and best specific combining crosses along with their per se performance as well as best heterotic crosses for different characters in okra

\begin{tabular}{|c|c|c|c|c|c|c|c|c|c|c|c|c|}
\hline \multirow{3}{*}{$\begin{array}{l}\text { Sr. } \\
\text { No. } \\
\\
1 .\end{array}$} & \multirow{3}{*}{$\begin{array}{l}\text { Traits } \\
\\
\text { DFF }\end{array}$} & \multicolumn{2}{|c|}{$\begin{array}{l}\text { Best performing } \\
\text { parents }\end{array}$} & \multicolumn{2}{|c|}{$\begin{array}{l}\text { Good general } \\
\text { combiners }\end{array}$} & \multicolumn{2}{|c|}{$\begin{array}{l}\text { Best cross per se } \\
\text { performance }\end{array}$} & \multicolumn{2}{|c|}{$\begin{array}{l}\text { Most heterotic cross over } \\
\text { check "JGOH-4" }\end{array}$} & \multicolumn{3}{|c|}{ Best specific crosses } \\
\hline & & \multirow{2}{*}{$\begin{array}{c}\text { Parents } \\
\text { Arka Abhay }\end{array}$} & \multirow{2}{*}{$\begin{array}{l}\text { Mean } \\
45.00\end{array}$} & \multirow{2}{*}{$\begin{array}{c}\text { Parents } \\
\text { Arka Abhay }\end{array}$} & \multirow{2}{*}{$\begin{array}{c}\begin{array}{c}\text { gca } \\
\text { effect }\end{array} \\
-0.66 * *\end{array}$} & & & & & \multirow{2}{*}{$\begin{array}{c}\text { Cross } \\
\text { NOL-18-12 x } \\
\text { NOL-18-14 }\end{array}$} & \multirow{2}{*}{$\begin{array}{c}\begin{array}{c}s c a \\
\text { effect }\end{array} \\
-3.28 * *\end{array}$} & \multirow{2}{*}{$\begin{array}{c}\text { Combination } \\
\text { Average x } \\
\text { Average }\end{array}$} \\
\hline & & & & & & $\begin{array}{l}\text { NOL-18-12 x } \\
\text { NOL-18-14 }\end{array}$ & 41.67 & $\begin{array}{l}\text { NOL-18-12 x } \\
\text { NOL-18-14 }\end{array}$ & $-10.71 * *$ & & & \\
\hline 2. & PH & NOL-18-14 & 92.80 & NOL-18-14 & $9.71 * *$ & $\begin{array}{l}\text { Arka Abhay x } \\
\text { GAO-5 }\end{array}$ & 96.73 & $\begin{array}{l}\text { Arka Abhay x } \\
\text { GAO-5 }\end{array}$ & $45.76 * *$ & $\begin{array}{l}\text { Arka Abhay x } \\
\text { GAO-5 }\end{array}$ & $15.16 * *$ & Good x Good \\
\hline 3. & $\mathrm{BPP}$ & Arka Abhay & 3.13 & NOL-18-14 & $0.33 * *$ & $\begin{array}{c}\text { Arka Abhay x } \\
\text { GAO-5, GAO- } \\
5 \text { x NOL-18-14 }\end{array}$ & 3.40 & $\begin{array}{c}\text { Arka Abhay x } \\
\text { GAO-5, GAO-5 } \\
\text { x NOL-18-14 }\end{array}$ & $37.84 * *$ & $\begin{array}{l}\text { NOL-18-9 x } \\
\text { NOL-18-16 }\end{array}$ & $0.51 * *$ & Average x Poor \\
\hline 4. & FW & NOL-18-14 & 15.83 & NOL-18-14 & $1.14 * *$ & $\begin{array}{c}\text { GAO-5 x NOL- } \\
18-14\end{array}$ & 16.72 & $\begin{array}{c}\text { GAO-5 x NOL- } \\
18-14\end{array}$ & $18.36 * *$ & $\begin{array}{l}\text { NOL-18-15 x } \\
\text { NOL-18-17 }\end{array}$ & $2.21 * *$ & Poor x Poor \\
\hline 5. & FL & NOL-18-14 & 13.55 & NOL-18-14 & $1.46^{* *}$ & $\begin{array}{c}\text { NOL-18-14 x } \\
\text { NOL-18-17 }\end{array}$ & 14.61 & $\begin{array}{c}\text { NOL-18-14 x } \\
\text { NOL-18-17 }\end{array}$ & $34.44 * *$ & $\begin{array}{l}\text { NOL-18-12 x } \\
\text { NOL-18-17 }\end{array}$ & $2.26^{* *}$ & Good x Average \\
\hline 6. & FD & NOL-18-14 & 1.65 & NOL-18-14 & $0.06 * *$ & $\begin{array}{l}\text { Arka Abhay x } \\
\text { GAO-5 }\end{array}$ & 1.68 & $\begin{array}{l}\text { Arka Abhay x } \\
\text { GAO-5 }\end{array}$ & $16.90 * *$ & $\begin{array}{l}\text { Arka Abhay x } \\
\text { GAO-5 }\end{array}$ & $0.14 * *$ & Average $\mathrm{x}$ Poor \\
\hline 7. & FPP & NOL-18-14 & 17.60 & NOL-18-14 & $1.69 * *$ & $\begin{array}{l}\text { Arka Abhay x } \\
\text { NOL-18-14 }\end{array}$ & 18.33 & $\begin{array}{c}\text { Arka Abhay x } \\
\text { NOL-18-14 }\end{array}$ & $19.05 * *$ & $\begin{array}{l}\text { NOL-18-9 x } \\
\text { NOL-18-16 }\end{array}$ & $2.79 * *$ & Poor x Poor \\
\hline 8. & FYPP & NOL-18-14 & 276.07 & NOL-18-14 & $\begin{array}{c}42.21 * \\
*\end{array}$ & $\begin{array}{c}\text { GAO-5 x NOL- } \\
18-14\end{array}$ & $\begin{array}{c}287.9 \\
0\end{array}$ & $\begin{array}{c}\text { GAO-5 x NOL- } \\
18-14\end{array}$ & $29.41 * *$ & $\begin{array}{l}\text { NOL-18-12 x } \\
\text { NOL-18-15 }\end{array}$ & $37.49 * *$ & Average $\mathrm{x}$ Poor \\
\hline 9. & IL & NOL-18-14 & 5.38 & NOL-18-14 & $-0.81 * *$ & $\begin{array}{c}\text { Arka Abhay x } \\
\text { GAO-5 }\end{array}$ & 4.29 & $\begin{array}{c}\text { Arka Abhay x } \\
\text { GAO-5 }\end{array}$ & $28.44 * *$ & $\begin{array}{l}\text { Arka Abhay x } \\
\text { GAO-5 }\end{array}$ & $-2.31 * *$ & Good x Poor \\
\hline 10. & NSPF & NOL-18-12 & 41.00 & NOL-18-17 & $-2.77 * *$ & $\begin{array}{l}\text { NOL-18-12 x } \\
\text { NOL-18-17 }\end{array}$ & 38.27 & $\begin{array}{l}\text { NOL-18-12 x } \\
\text { NOL-18-17 }\end{array}$ & -9.32 & $\begin{array}{c}\text { GAO-5 x } \\
\text { NOL-18-15 }\end{array}$ & $-8.92 * *$ & $\begin{array}{c}\text { Average } \mathrm{x} \\
\text { Average }\end{array}$ \\
\hline 11. & HSW & NOL-18-9 & 3.87 & NOL-18-9 & $-0.39 * *$ & $\begin{array}{c}\text { NOL-18-12 x } \\
\text { NOL-18-16 }\end{array}$ & 3.70 & $\begin{array}{c}\text { NOL-18-12 x } \\
\text { NOL-18-16 }\end{array}$ & $-15.91 * *$ & $\begin{array}{c}\text { NOL-18-12 x } \\
\text { NOL-18-16 }\end{array}$ & $-0.79 * *$ & Average x Good \\
\hline
\end{tabular}

* - Significant at $5 \%$ and **-Significant at $1 \%$

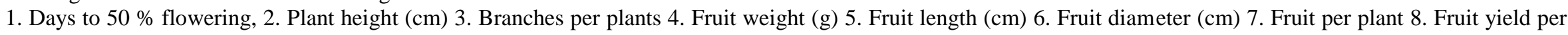
plant 9. Internodal length 10 . Number of seeds per fruit 11.100 seed weight 
Similar result was reported by Lyngdoh et al., (2017), Shwetha et al., (2018), Demta and Surin (2019) and Pithiya et al., (2020). A summarized account of the best parents, per se performance best general combiners, best heterotic crosses and best specific combinations revealed that the best performing parents may be a best general combiner. Further, the best general combiner or best parents per se may not always produce best specific combinations for all the characters. Therefore, it is more desirable to select crosses based on the per se performance rather than magnitude of sca effects. Similar result has been earlier reported by Patel et al., (2015b), More et al., (2017), Satish et al., (2017), Lokeswari et al., (2018) and Demta and Surin (2019). The cross showing low sca effects may exhibit high per se performance also. Similar result has been reported by Patel et al., (2015b), Lyngdoh et al., (2017) and Hadiya et al., (2018). From the top six hybrids, three hybrids viz., GAO-5 x NOL-18-14, Arka Abhay $x$ NOL-18-14 and Arka Abhay $x$ GAO-5 manifested high heterosis coupled with high and significant $s c a$ effects for fruit yield per plant, while three hybrids viz., NOL18-14 x NOL-18-15, NOL-18-14 x NOL-1817 and NOL-18-12 x NOL-18-14 exhibited high heterosis coupled with non-significant sca effect.

The hybrids exhibiting high per se performance for fruit per plant result from average $\mathrm{x}$ average, average $\mathrm{x}$ good and good $\mathrm{x}$ poor hybrid combinations. It is interesting to note that the average general combining parents when crossed do not always produce high sca effects; likewise poor general combining parents did not always produce low sca effects. Similar results have been reported by Patel et al., (2015), Lyngdoh et al., (2017), More et al., (2017), Satish et al., (2017), Hadiya et al., (2018), Gavint et al., (2018) and Kayande et al., (2018).
Appraisal of data (Table 4) revealed that for most of the characters; hybrids exhibiting higher sca effects for fruit yield and yield contributing characters involved poor $\mathrm{x}$ poor, average $\mathrm{x}$ poor, good $\mathrm{x}$ poor and average $\mathrm{x}$ average combiner parents, indicating the presence of both additive and non-additive gene effects for controlling fruit yield and its contributing characters. These results are in agreement with Shwetha et al., (2018), Demta and Surin (2019) and Pithiya et al., (2020). The hybrids viz., NOL-18-15 x NOL-18-17 and NOL-18-9 x NOL-18-16 showed good sca effects with involved poor $\mathrm{x}$ poor combining parents indicating over dominance and epistatic interactions. This may be due to genetic diversity in the form of heterozygous loci.

Marked negative and significant sca effects in crosses between average $\mathrm{x}$ good, average $\mathrm{x}$ average and good $\mathrm{x}$ poor combiners could be attributed to the lack of co-adaptation between favourable alleles of the parents involved. Whereas marked positive and significant $s c a$ effects in crosses between poor $\mathrm{x}$ poor, good $\mathrm{x}$ good, average $\mathrm{x}$ poor and good $x$ average general combiners could be ascribed to better complementation between favourable alleles of the parents involved [Patel and Mehta (1985)].

The sca effect represents dominance effect and can be related with heterosis. The correlation between per se performance and the sca effect of hybrids for majority of the characters indicated that $s c a$ effects of a cross can reasonably be predicted from per se performance. However, the inspection of sca effects and mean performance of individual crosses indicated that the crosses having high sca effects did not always possess high mean (Table 4).

However, the crosses involving high sca effects did not always involve parents with 
high gca effects, thereby, suggesting the presence of intra-allelic gene interactions. The sca effects of certain crosses in the undesirable direction could be due to the failure of desirable alleles of the parents to co-operate. As a result, a cross from good general combiner parents may exhibit poor sca effects. Table 4 revealed that there was some degree of correspondence between per se performance and sca effects of hybrids as well as gca effects of parents and estimates of heterosis for most of characters. Hence, gca and sca effects and per se performance all play a role in manifestation of heterosis for various characters. From the about discussion it is clear that hybrids NOL-18-12 x NOL-1814 (for days to $50 \%$ flowering), Arka Abhay $\mathrm{x}$ GAO-5 (for plant height and fruit diameter and internodal length), NOL-18-12 x NOL18-16 (for 100 seed weight) exhibited higher gca and sca effects with high mean performance.

\section{References}

Anonymous(2019).https://www.indiastat.com/ table/agriculturedata/2/la-dys-fingerokra/23059/14905/data.aspx.

Demta, S. S. and Surin, S. S. (2019). Combining ability analysis for yield and its characters of okra crosses [Abelmoschus esculentus (L.) Moench]. J. Pharma. Phyto., 2: 436-439.

Devi, S., Choudhary, B. and Verma, I. (2017). Combining ability analysis for yield and yield contributing characters in okra [Abelmoschus esculentus (L.) Moench]. The Bioscan, 12(3): 1593-1596.

Gavint, K. N., Vadodariya, K. V. and Bilwal, B. B. (2018b). Combining ability analysis for fruit yield and its attributing traits in okra [Abelmoschus esculentus (L.) Moench]. Int. J. Curr. Microbiol. Appl. Sci., 7(2): 1821-1828.

Hadiya, D. N., Mali, S. C., Gamit, A. M. and Sangani, J. L. (2018). Combining ability studies in okra [Abelmoschus esculentus (L.) Moench]. J. Pharma. Phyto., 7(5): 2525-2528.

Kayande, N.V., Kumbhalkar, H. B. and Shinde, S. (2018). Selection of parents based on combining ability studies in okra [Abelmoschus esculentus L. Moench]. Int. J. Curr. Microbiol. Appl. Sci., 6: 1935-1940.

Kumar, R., Shanthakumar, G., Kamatar, M., Patil, V. and Adiger, S. (2016). Combining ability studies in newly derived inbred lines of okra [Abelmoschus esculentus (L.) Moench]. Adv. Life Sci., 5(23): 11105-11108.

Lokeswari, S., Ashok, P. and Kala, R. (2018). Studies on combining ability and gene action for yield and yield contributing characters in okra [Abelmoschus esculentus (L.) Moench]. J. Pharma. Phyto., 7(6): 2103-2106.

Lyngdoh, Y. A., Mulge, R., Shadap, A., Singh, J. and Sangwan, S. (2017). Combining ability analysis in near homozygous lines of okra [Abelmoschus esculentus (L.) Moench] for yield and yield attributing parameters. J. Appl. Natural Sci., 9(1): 324-331.

More, S. J., Chaudhari, K. N., Vaidya, G. B. and Chawla, S. L. (2017b). Multienvironment analyses of genetic components and combining abilities in relation to heterosis in okra [Abelmoschus esculentus (L.) Moench]. Int. J. Curr. Microbiol. Appl. Sci., 6(12): 2835-2842.

Makdoomi, M. I., Wani, K. P., Khan, S. H., Bhat, M. A., Hussain, K., Afroza, B. and Nabi, A. (2018). Combining ability analysis in okra [Abelmoschus esculentus (L.) Moench]. J. Pharma. Phyto., 7(2): 460-465.

Patel, H. B., Bhanderi, D. R., Patel, A. I., Tank, R. V. and Kumar, A. (2015b). Combining ability analysis for yield and yield components in okra [Abelmoschus 
esculentus (L.) Moench]. Trends Biosci., 8(9): 2240-2245.

Patel, U.G and Mehta, N.P. (1985). Studies on heterosis, combining ability and stability performance in cotton $(G$. herbaceum L.). Indian J. Genet., 37: 4548

Patil, S. S., Patil, P. P., Lodam, V. A. and Desai, D. T. (2016). Evaluating genotypes for combining ability through diallel analysis in okra over different environments. Ele. J. Pl. Br.,7(3), 582588.

Paul,T., Desai,R.T. and Choudhary,R. (2017b). Genetic architecture, combining ability and gene action study in okra [Abelmoschus esculentus (L.) Moench]. Int. J. Curr. Microbiol. Appl. Sci., 6(4): 851-858.

Pithiya, D. J., Jethava, A. S., Zinzala, S. N. and Vachhani, J. H. (2020). Study on combining ability in okra [Abelmoschus esculentus (L.) Moench.]. Int. J. Chem. Stud., 8(1): 676-679.

Rameshkumar, D., Gunasekar, R. and Sankar, R. (2017). Gene action studies for quantitative and qualitative traits in okra [Abelmoschus esculentus (L.) Moench]. Int. J. Chem. Stud., 5(5): 2309-2312.

Satish, K., Suresh, K., Agalodiya, A. V. and
Prajapati, D. B. (2017). Combining ability for yield and its attributing traits in okra [Abelmoschus esculentus (L.) Moench]. Int. J. Curr. Microbiol. Appl. Sci., 6(9): 1944-1954.

Shwetha, A., Mulge, R., Evoor, S., Kantharaju, V. and Masuti, D. A. (2018). Diallel analysis for combining ability studies in okra [Abelmoschus esculentus (L.) Moench] for yield and quality parameters. Int. J. Curr. Microbiol. Appl. Sci., 7(9): 2114-2121.

*Sprague, G.F. and Tatum, L.A. (1942). General versus specific combining ability in single crosses in corn. Agronomy, 34: 923-932.

Tiwari, J. N., Kumar, S. and Ahlawat, T. R. (2016). Combining ability studies for various horticultural traits in okra [Abelmoschus esculentus (L.) Moench] under south-Gujarat conditions. J. Farm Sci., 29(1): 53-56.

Wakode, M. M., Bhave, S. G., Navhale, V. C., Dalvi, V. V., Devmore, J. P. and Mahadik, S. G. (2016). Combining ability studies in okra [Abelmoschus esculentus (L.) Moench]. Ele. J. Pl. Br., 7(4): 1007-1013.

\section{How to cite this article:}

Koli, H. K. and Patel, A. I. 2020. Combining Ability Analysis for Yield and Yield Components in Okra [Abelmoschus esculentus (L.) Moench]. Int.J.Curr.Microbiol.App.Sci. 9(10): 665-673. doi: https://doi.org/10.20546/ijcmas.2020.910.080 